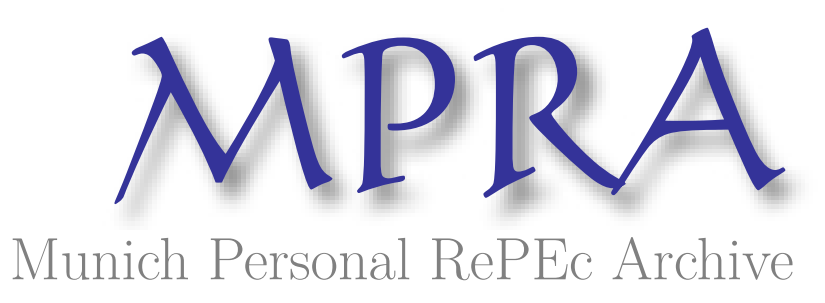

\title{
Variance Ratio Testing for Fractional Cointegration in Presence of Trends and Trend Breaks
}

\author{
Dechert, Andreas
}

4 September 2012

Online at https://mpra.ub.uni-muenchen.de/41044/

MPRA Paper No. 41044, posted 05 Sep 2012 14:04 UTC 


\title{
Variance Ratio Testing for Fractional Cointegration in Presence of Trends and Trend Breaks
}

\author{
Andreas Dechert* \\ Julius-Maximilians-Universität Würzburg \\ Version: 3rd September, 2012
}

\begin{abstract}
Modeling fractional cointegration relationships has become a major topic in applied time series analysis as it steps back from the traditional rigid $I(1) / I(0)$ methodology. Hence, the number of proposed tests and approaches has grown over the last decade. The aim of this paper is to study the nonparametric variance ratio approach suggested by Nielsen for the case of fractional cointegration in presence of linear trend and trend breaks. The consideration of trend breaks is very important in order to avoid spurious fractional integration, so this possibility should be regarded by practitioners. This paper proposes to calculate p-values by means of gamma distributions and gives response regressions parameters for the asymptotic moments of them. In Monte Carlo simulations this work compares the power of the approach against a Johansen type rank test suggested, which is robust against trend breaks but not fractional (co-)integration. As the approach also obtains an estimator for the cointegration space, the paper compares it with OLS estimates in simulations. As an empirical example the validity of the market expectation hypothesis is tested for monthly Treasury bill rates ranging from 1958-2011, which might have a trend break around September 1979 due to change of American monetary policy.
\end{abstract}

JEL-Classification: C32, C14, E43

Keywords: fractional integration, fractional cointegration, long memory, variance ratio, nonparametric, trend breaks, market expectation hypothesis

*Department of Economics, Email: andreas.dechert@uni-wuerzburg.de 


\section{Introduction}

The use of cointegration has reached the rank of a standard econometric tool since the seminal work of Engle and Granger (1987). This method has the great advantage that nonstationary unit root process can be examined without differencing. The existence of a cointegration relations leads to the interpretation that there is stationary equilibrium between nonstationary time series, which is attractive for empirical research in economics and especially in finance. In this view, it seems to be natural, that the concept of fractional cointegration has attracted more attention than it can be seen as a generalization of the standard cointegration concept. The fractional methodology has the great advantage, that the integration order is not stucked to an integer digit anymore and opens up a very wide range of modeling empirical specifics like long range dependencies. This leads to the interpretation of fractional integrated processes for long memory property as the coefficients of an infinite moving average representation is decaying in a hyperbolic way. It should be regarded, that for most time series, the hypothesis $d=1$ can't be rejected even if fractional integration is taken into account. The concept is more interesting to model cointegration error processes as it shows the persistence of exogenous shocks to a system of time series. The possibility of fractional integrated errors can also be seen as a reason for rejecting cointegration in classical approaches.

The consideration of deterministic components is important as it affects the distribution of the most test statistics and also the consistency of integration order estimating. The allowance for breaks in deterministic processes goes back to the seminal work of Perron (1989), who founds, that the negligence of breaks leads to over-rejection of stationarity. In the fractional literature Sibbertsen (2004) mentioned, that changes in the deterministic components might endow spurious long memory as it causes additional persistence if it is not regarded. Therefore, the possibility of breaks should be taken into account by practitioners.

An interesting approach dealing with fractional cointegration was proposed by Nielsen (2010) who considered nonparametric Variance Ratio (hereinafter: VR) tests for the cointegration rank. The idea of the test was already written in Nielsen (2008) in the univariate case and was thought as nonparametric unit root test. The test could also been adopted in the fractional context as a test for the integration parameter $d$, as it doesn't assume the knowledge of $d$ to calculate the test statistic. As the nature of the test is nonparametric, there is no need to specify the autocorrelation structure like in the Dickey-Fuller type tests with a lag parameter or a bandwidth parameter like in the most existing fractional cointegration methods, e.g. Nielsen and Shimotsu (2007) or Shimotsu 
(2010). Another advantage of the approach is, that there is no need to estimate the cointegration relationship itself, but gives the possibility of a consistent estimator of the cointegration space.

Through simulations we determine the moments of the VR statistic according to the dimension of the examined system $p$ and its hypothetical cointegration rank $r$. For these we give response regression parameters to calculate critical values and p-values using gamma distributions. In Monte Carlo simulation we show first the effects of neglecting a trend break in cointegration analysis. Furthermore, we compare the VR approach in broken trend situations with Johansen type rank test proposed by Johansen, Mosconi and Nielsen (hereinafter JMN: 2000), which takes also trend breaks into account, but neglects the possibility of fractional (co-)integration. As the VR approach also delivers a consistent estimator for the cointegration space, we compare it also with OLS estimates under fractional cointegration.

In empirical economic analysis, cointegration is applied in many fields to prove theoretical models e.g. the market expectation hypothesis. It states that nonstationary interest rates with different maturities incorporate a stationary equilibrium as Campbell and Shiller (1987) imposed. Hence, we choose monthly Treasury bill rates ranging from December 1958 to December 2011. The rates are supposed to have a trend break in September 1979 due to the regime switch in US monetary policy and we wish to take this into account in our analysis.

The paper is organized as follows: the following section introduces first the fractional integration methodology and the VR testing. Then estimated response surface parameters for critical values are presented and some Monte Carlo simulations are conducted to show power of the test and benefits of the approach in the third section. The fourth section demonstrates an empirical application of the approach to test the implications of the market expectation hypothesis for Tbill rates and finally the paper is closed with a conclusion.

\section{Methodology}

As a first step we want to introduce the concept of fractional integration. Consider we observe a time series $y_{t}$, which is integrated of a non-integer order $d$. Then we can motivate 
an infinite moving average process through a power series expansion

$$
\begin{aligned}
y_{t} & =\Delta^{-d} \varepsilon_{t}=\varepsilon_{t}+d \varepsilon_{t-1}+\frac{d(d+1)}{2 !} \varepsilon_{t-2}+\frac{d(d+1)(d+2)}{3 !} \varepsilon_{t-3}+\ldots \\
& =\sum_{k=0}^{t} \frac{\Gamma(d+k)}{\Gamma(d) \Gamma(k+1)} \cdot \varepsilon_{t-k}
\end{aligned}
$$

We assume $d>1 / 2$ for the process $y_{t}$, which means that the process is not stationary. Then we can formulate its fractional partial sum

$$
\tilde{y}_{t}=\Delta^{-d_{1}} y_{t}=\Delta^{-\left(d+d_{1}\right)} \varepsilon_{t} \quad \text { for } \quad d_{1}>0 \text { and } \quad t=1, \ldots, T
$$

Under some regularity conditions on the error term $\varepsilon_{t}$ and for $d>1 / 2$, a fractional functional central limit theorem can be obtained for $y_{t}$ and analogous for $\tilde{y}_{t}$

$$
T^{1 / 2-d} y_{\lfloor s T\rfloor} \Rightarrow \sigma_{y} W_{d}(s), \quad 0<s \leq 1
$$

From this theorem, a limit for $T \rightarrow \infty$ on the second uncentered moment of $y_{t}$ and $\tilde{y}_{t}$ can be stated with

$$
T^{2 d} \sum_{t=1}^{T} y_{t}^{2} \stackrel{D}{\rightarrow} \sigma_{y}^{2} \int_{0}^{1} W_{d}(s)^{2} d s \quad \text { and } \quad T^{2 d+d_{1}} \sum_{t=1}^{T} \tilde{y}_{t}^{2} \stackrel{D}{\rightarrow} \sigma_{y}^{2} \int_{0}^{1} W_{d+d_{1}}(s)^{2} d s
$$

Dividing these moments yields a variance ratio test statistic

$$
\rho\left(d_{1}\right)=T^{2 d_{1}} \frac{\sum_{t=1}^{T} y_{t}^{2}}{\sum_{t=1}^{T} \tilde{y}_{t}^{2}} \stackrel{D}{\rightarrow} \frac{\int_{0}^{1} W_{d}(s)^{2} d s}{\int_{0}^{1} W_{d+d_{1}}(s)^{2} d s}
$$

which is free from the nuisance parameter $\sigma_{y}^{2}$, which could be left out estimating. Only the parameter $d_{1}$ has to be specified and indexes the family of the test. Nielsen (2008) found out through simulations, that the choice $d_{1}=0.1$ yields highest power of the test. Another also interesting choice would be $d_{1}=1$, because then the test is equivalent to Breitung's statistic (2002) to test for a unit root against nonlinear alternatives.

Nielsen (2010) extended the variance ratio test for multivariate $p$-vector time series $\boldsymbol{y}_{t}$ in purpose to test the presence of fractional cointegration, which is a generalization of the classical cointegration concept defined by Engle and Granger (1987). Consider we observe the following triangular system of time series

$$
\begin{aligned}
\Delta^{d-b}\left(\boldsymbol{y}_{1 t}-\gamma^{\prime} \boldsymbol{y}_{2 t}\right) & =\varepsilon_{1 t} \\
\Delta^{d} \boldsymbol{y}_{2 t} & =\varepsilon_{2 t}, \quad t=1, \ldots, T
\end{aligned}
$$


supposing that all $p$ components of $\boldsymbol{y}_{t}=\left\{\boldsymbol{y}_{1 t}, \boldsymbol{y}_{2 t}\right\}^{\prime}$ are sharing the same order of integration $d$ and all $r$ cointegration relations the same strength of cointegration $b$. The $r$-dimensional vector $\boldsymbol{y}_{1 t}$ forms $r$ number of cointegration relations integrated by order $d-b$ with the $p-r$-dimensional vector $\boldsymbol{y}_{2 t}$, which contains the stochastic trends integrated of order $d$ driving the system. It should be noted, that we have only (fractional) cointegration when the cointegration strength parameter $b>0$. When we set $d=b=1$ we have the classical cointegration setup defined by Engle and Granger (1987) and hence, fractional cointegration is a generalization of classical cointegration. Alternatively, a cointegrated system can be represented with the cointegration relations yielding with $\boldsymbol{\beta}^{\prime} \boldsymbol{y}_{t}$ where $\boldsymbol{\beta}$ is the cointegration space matrix defined as $\boldsymbol{\beta}=\left(\boldsymbol{I}_{r},-\gamma\right)^{\prime}$. The error terms $\boldsymbol{\varepsilon}_{1 t}$ and $\varepsilon_{2 t}$ are integrated of order zero and might be autocorrelated of an unknown structure with zero mean.

Defining the matrices $\boldsymbol{A}_{T}=\sum_{t=1}^{T} \boldsymbol{y}_{t} \boldsymbol{y}_{t}^{\prime}$ and $\boldsymbol{B}_{T}=\sum_{t=1}^{T} \tilde{\boldsymbol{y}}_{t} \tilde{\boldsymbol{y}}_{t}^{\prime}$ we can calculate analogously to the univariate the statistic

$$
\boldsymbol{R}_{T}\left(d_{1}\right)=\boldsymbol{A}_{T} \boldsymbol{B}_{T}^{-1}
$$

Let $\lambda_{1} \leq \lambda_{2} \leq \ldots \leq \lambda_{p}$ be the ascending ordered eigenvalues of $\boldsymbol{R}_{T}\left(d_{1}\right)$ and $\boldsymbol{\eta}_{1}, \boldsymbol{\eta}_{2}, \ldots, \boldsymbol{\eta}_{p}$ the according eigenvectors resulting from solving the eigenproblem

$$
\left|\lambda \boldsymbol{B}_{T}-\boldsymbol{A}_{T}\right|=0
$$

These are used to calculate the nonparametric variance ratio trace statistic

$$
\Lambda_{p-r}\left(d_{1}\right)=T^{2 d_{1}} \sum_{i=1}^{p-r} \lambda_{i}, \quad r=0,1, \ldots, p-1
$$

The distribution of the test statistic (9) depends on the presence of deterministic components. Consider the processes in $\boldsymbol{y}_{t}$ are generated by the following data generating process

$$
y_{t}=\mu_{0}+\mu_{1} \cdot t+\mu_{2} \cdot D T_{t}+\Delta^{-d} \varepsilon_{t}
$$

where $D T_{t}=\sum_{i=1}^{t} D U_{i} \quad$ with $D U_{i}= \begin{cases}1 & \text { when } i>T_{b} \\ 0 & \text { else. }\end{cases}$

and $T_{b}=\tau T$ a possible break-date of the trending component of $y_{t}$. This kind of deterministic process was proposed by Perron (1989) and was named the changing growth model corresponding to the break in the slope of the trending component. Nielsen (2010) was able to establish the asymptotic distribution of the VR statistic by fractional Brownian motions, possibly detrended or demeaned. In line with his work we assume, that 
$\Lambda_{p, r}\left(d_{1}, \tau\right)$ is distributed for $T \rightarrow \infty$ by

$$
\Lambda_{p-r, \tau}\left(d_{1}\right) \stackrel{D}{\rightarrow} \operatorname{tr}\left[\int_{0}^{1} \boldsymbol{B}_{\tau, d}^{p-r}(s) \boldsymbol{B}_{\tau, d}^{p-r}(s)^{\prime} d s\left(\int_{0}^{1} \boldsymbol{B}_{\tau, d+d_{1}}^{p-r}(s) \boldsymbol{B}_{\tau, d+d_{1}}^{p-r}(s)^{\prime} d s\right)^{-1}\right]
$$

where $B_{\tau, d}$ denotes a fractional Brownian motion integrated of order $d$ and corrected by a broken trend with break in $\tau$ according to the assumed DGP in (10).

A consistent estimator of the cointegration space $\boldsymbol{\beta}$, when the system $\boldsymbol{y}_{t}$ is cointegrated of rank $r$ can be formulated with $\boldsymbol{\eta}(r)=\left(\boldsymbol{\eta}_{p-r}, \ldots, \boldsymbol{\eta}_{r}\right)$, which is $p \times r$ matrix of the eigenvectors $\boldsymbol{\eta}_{i}$ belonging to $r$ biggest eigenvalues. The estimator is then

$$
\hat{\boldsymbol{\beta}}_{r}=\boldsymbol{\eta}(r)\left[\left(\boldsymbol{I}_{r}, \mathbf{0}_{r \times(p-r)}\right) \cdot \boldsymbol{\eta}(r)\right]^{-1}
$$

with $\mathbf{0}_{m \times n}$ as a $m \times n$-dimensional null matrix. Nielsen (2010) also shows that the estimator is consistent in the sense that the angle between the true and the estimated space converges to zero asymptotically, but the framework gives no closed distribution and, hence, the estimated space can't be tested against theoretical assumptions.

\section{Critical Values and Simulations}

Since the asymptotic distribution in (12) has no analytical closed form it is necessary to obtain critical values for the statistic (9) through Monte Carlo simulations in dependence of $d$ and $\tau$. In figure 1 we have the estimated density of the VR statistic for $d=1$ and $\tau=0.5$ and compare it with a approximated gamma distribution, which indicates a moderate fit. When we focus on the relevant critical quantiles of the statistic in figure 2 , then we have a quite good fit. The approximation gets worse while $d$ decreases, but it is still acceptable as later Monte Carlo simulations show. Hence, we decide to approximate the test distribution with gamma distributions and model the relevant first two moments of these instead of approximating the critical quantiles.

Figures 3 and 4 show the structure of the moments of simulated VR statistic for $p-r=1$ in dependence of $\tau$ and $d$. It can be seen that the moments are symmetric around $\tau=0.5$ which allows us to abstain simulations for $\tau>0.5$. When we observe a trend break at $\tau$, then we can divide the sample in two parts with relative sample duration $\tau$ and $1-\tau$. Hence, let $v$ be the smallest length of these and we use this parameter for possible response regressions for the moments of approximated test distribution. For these we choose polynomials of $d, v$ and $T$ and have the form for each moment and situation $p-r$. 


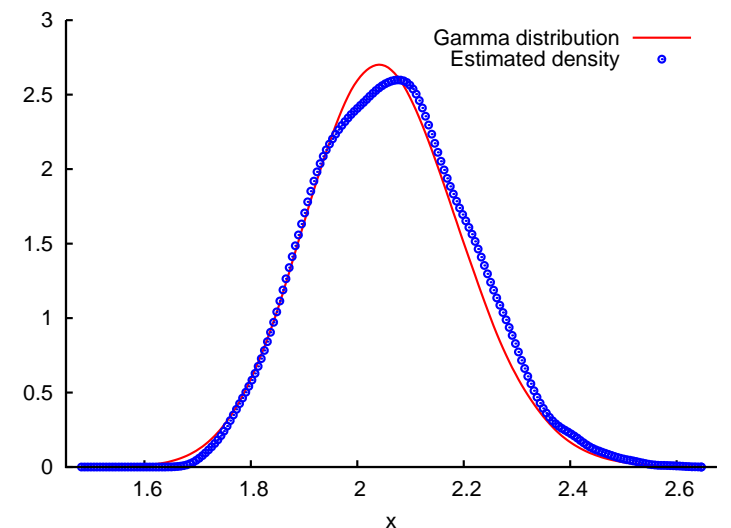

Figure 1: Density of VR statistic for $\tau=$ 0.5 and $d=0.8$

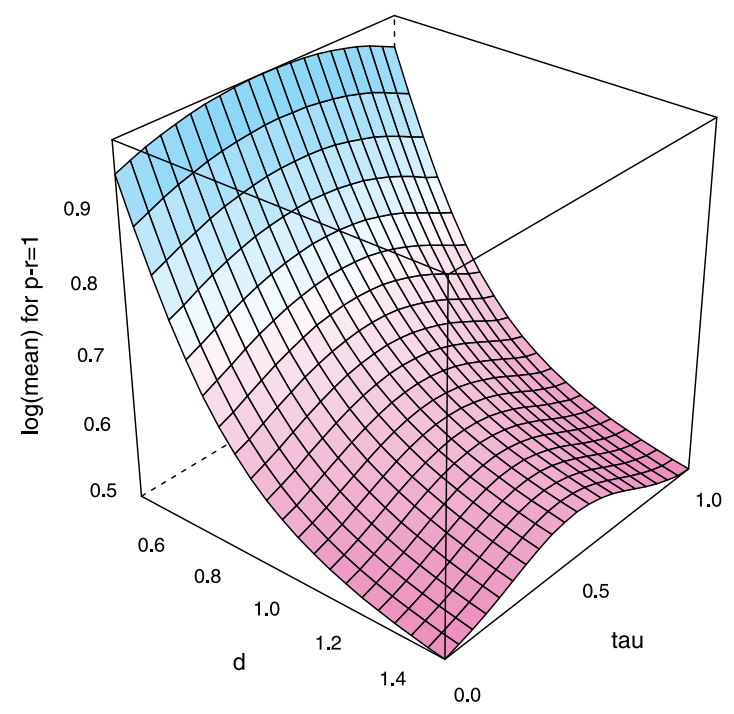

Figure 3: Simulated $\log ($ mean $)$

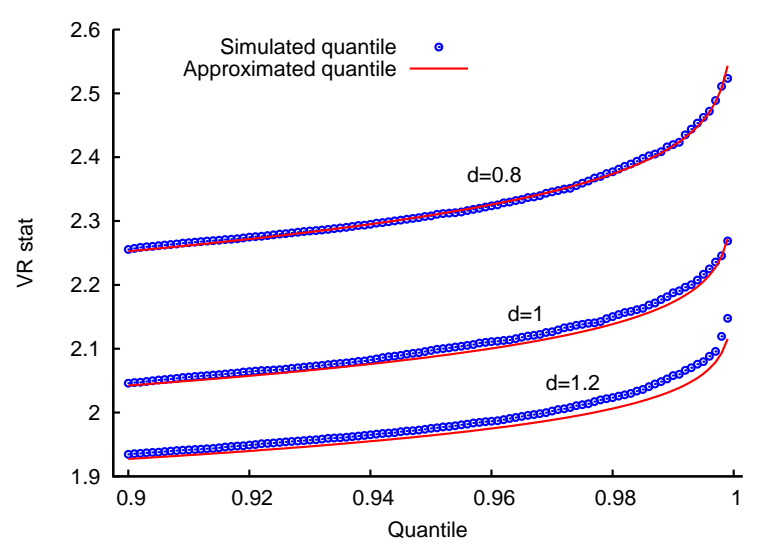

Figure 2: Critical quantiles of VR statistic

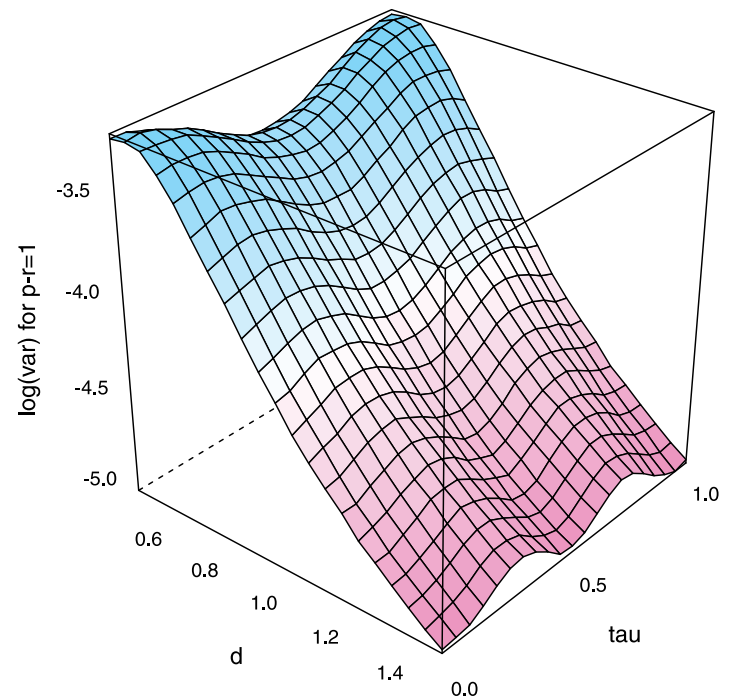

Figure 4: Simulated $\log (\operatorname{var})$

This type of modeling of the moments bases on Doornik (1998) and JMN (2000), who did response regressions for related cointegration trace and eigenvalue statistics. We deviate from their work by modeling the moments for each $p-r$ separately, because we could not find any adequate fitting equation.

$$
\log \left(\text { moment }_{p-r}\right)=\sum_{i=0}^{9} \sum_{j=0}^{6} \sum_{k=0}^{3} \delta_{i j k} d^{i} v^{j} T^{-k}+\epsilon_{\text {moment }, p-r}
$$

This model sums up to 162 regressors for 1848 observations for each simulated moment and situation $p-r$. The moments were simulated for $p-r=1, \ldots, 8$ and various values of $d, v$ and $T$. The setup was as follows: 


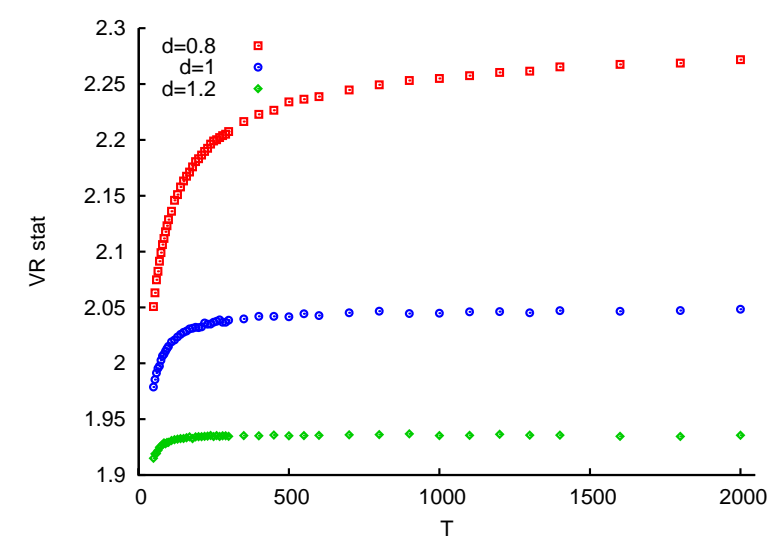

Figure 5: 5\% critical values in dependence of simulated sample size

- $d \in[0.5,0.55, \ldots, 1.45,1.5]$

- $v \in[0,0.05, \ldots, 0.45,0.5]$

- $T=\operatorname{int}(500 / j) \quad$ for $j=1, \ldots, 8$

Each simulation was replicated 100.000 times. Afterwards regressions for the moments according to equation (14) were conducted by using ordinary least squares. The number of regressors could be reduced by sequential elimination of insignificant variables. The asymptotic moments were then computed by letting $T \rightarrow \infty$. The estimated coefficients $\hat{\delta}_{i j 0}$ are reported in table 1 and 2 .

Figure 5 illustrates that there is a small sample problem for the VR approach, when $d$ is rather low. In this figure we have simulated $5 \%$ critical values in dependence of sample size $T$ and for $d=0.8,1$ and 1.2. Especially for the case of $d=0.8$ we have hardly any asymptotic convergence. For the other cases $d=1$ and $d=1.2$ there is a convergence already with smaller sample sizes like $T=250$ and so we might expect that the VR approach performs better than with rather low values of $d$. Thereby, we consider also bootstrapping as a way to determine $p$-values.

For the following Monte Carlo studies we consider a bivariate cointegration system of the form

$$
\begin{aligned}
& y_{1, t}=\mu_{1} t+\mu_{2} D T_{t}+\Delta^{-d} u_{1 t} \\
& y_{2, t}=y_{1, t}+\Delta^{-(d-b)} u_{2 t}
\end{aligned}
$$

with the broken trend variable $D T_{t}$ defined in equation (11) and two different types of 
Table 1: Estimated response surface parameters for $\log ($ mean)

\begin{tabular}{c|cccccccc}
\hline$p-r$ & 1 & 2 & 3 & 4 & 5 & 6 & 7 & 8 \\
\hline Constant & 0.8329 & 1.5440 & 1.9725 & 2.2754 & 2.5089 & 2.7025 & 2.8657 & 3.0067 \\
$d$ & 4.4911 & 4.2643 & 4.0481 & 3.8745 & 3.7473 & 3.6194 & 3.5052 & 3.4021 \\
$d^{2}$ & -16.844 & -16.004 & -15.315 & -14.706 & -14.250 & -13.798 & -13.376 & -12.980 \\
$d^{3}$ & 23.710 & 22.494 & 21.649 & 20.842 & 20.267 & 19.686 & 19.123 & 18.579 \\
$d^{4}$ & -16.682 & -15.800 & -15.324 & -14.813 & -14.486 & -14.138 & -13.783 & -13.425 \\
$d^{5}$ & 5.8981 & 5.5762 & 5.4645 & 5.3139 & 5.2366 & 5.1433 & 5.0391 & 4.9256 \\
$d^{6}$ & -0.8376 & -0.7901 & -0.7847 & -0.7690 & -0.7651 & -0.7572 & -0.7463 & -0.7327 \\
$v^{2}$ & -0.2481 & -0.1517 & -0.0794 & -0.0208 & 0.0253 & 0.0650 & 0.0980 & 0.1261 \\
$v^{3}$ & 0.7745 & 0.4886 & 0.2795 & 0.1269 & 0.0195 & -0.0605 & -0.1212 & -0.1689 \\
$v d^{2}$ & 1.1609 & 1.0554 & 0.9782 & 0.9237 & 0.8892 & 0.8646 & 0.8473 & 0.8342 \\
$v d^{3}$ & -2.0533 & -1.7687 & -1.5599 & -1.4138 & -1.3247 & -1.2613 & -1.2184 & -1.1883 \\
$v d^{4}$ & 1.2655 & 1.0294 & 0.8592 & 0.7453 & 0.6820 & 0.6401 & 0.6151 & 0.6005 \\
$v d^{5}$ & -0.2629 & -0.2027 & -0.1597 & -0.1319 & -0.1177 & -0.1092 & -0.1049 & -0.1032 \\
$v^{2} d$ & 1.0570 & 0.8054 & 0.5914 & 0.4051 & 0.2461 & 0.1025 & -0.0237 & -0.1352 \\
$v^{2} d^{4}$ & -0.4044 & -0.1555 & 0.0186 & 0.1246 & 0.1817 & 0.2120 & 0.2248 & 0.2277 \\
$v^{2} d^{5}$ & 0.1178 & 0.0266 & -0.0368 & -0.0744 & -0.0937 & -0.1033 & -0.1065 & -0.1062 \\
$v^{3} d$ & -5.5378 & -4.2837 & -3.2482 & -2.4724 & -1.9049 & -1.4764 & -1.1464 & -0.8844 \\
$v^{3} d^{2}$ & 3.5405 & 2.3113 & 1.3566 & 0.7293 & 0.3551 & 0.1485 & 0.0499 & 0.0174 \\
$v^{4} d$ & 3.3883 & 2.9412 & 2.4664 & 2.0981 & 1.8187 & 1.6132 & 1.4586 & 1.3409 \\
$v^{4} d^{2}$ & -2.8170 & -1.9794 & -1.2480 & -0.7571 & -0.4617 & -0.3101 & -0.2528 & -0.2554 \\
\hline$R^{2}$ & 0.99988 & 0.99988 & 0.99987 & 0.99986 & 0.99985 & 0.99984 & 0.99983 & 0.99982 \\
$\hat{\sigma}_{\epsilon}$ & 0.00099 & 0.00095 & 0.00092 & 0.00089 & 0.00088 & 0.00087 & 0.00087 & 0.00086 \\
\hline & & & & & & & &
\end{tabular}

error terms

Type A: $u_{1, t}, u_{2, t} \sim \operatorname{IID}(0,1)$

Type B: $\quad u_{j, t}=0.5 u_{j, t-1}+\varepsilon_{j, t} \quad$ with $\quad \varepsilon_{j, t} \sim \operatorname{IID}(0,1) \quad$ for $\quad j=1,2$.

The conducted Monte Carlo studies are two folds. First we examine the case, where there is actually no cointegration (i.e. $b=0$ ) relation between $y_{1, t}$ and $y_{2, t}$, but they incorporate a trend break at the relative break location $\tau$. We assume throughout the study that $\mu_{1}=0$, which means that we have no trending behavior until $\tau$ and afterwards a linear trend with slope $\mu_{2}$. The decision $\mu_{1} \neq 0$ doesn't change the results at all and is therefore arbitrary. Now we want to apply the VR approach on the two not cointegrated processes with the two assumptions:

1. there is no trend and detrend both series linearly (LT) 
Table 2: Estimated response surface parameters for $\log ($ var $)$

\begin{tabular}{c|cccccccc}
\hline$p-r$ & 1 & 2 & 3 & 4 & 5 & 6 & 7 & 8 \\
\hline Constant & -5.8240 & -5.2782 & -4.9958 & -4.8915 & -4.7755 & -4.6879 & -4.5715 & -4.5925 \\
$d$ & 2.0742 & 3.8523 & 5.2889 & 7.3369 & 8.2834 & 9.1756 & 10.370 & 11.459 \\
$d^{2}$ & 37.060 & 28.627 & 21.685 & 12.332 & 7.9363 & 3.6878 & -3.3627 & -7.6987 \\
$d^{3}$ & -104.01 & -88.392 & -75.450 & -57.849 & -50.124 & -42.565 & -28.044 & -20.692 \\
$d^{4}$ & 108.40 & 94.230 & 82.390 & 65.960 & 59.386 & 52.956 & 38.750 & 32.655 \\
$d^{5}$ & -51.012 & -44.728 & -39.443 & -31.929 & -29.217 & -26.595 & -19.865 & -17.426 \\
$d^{6}$ & 9.1235 & 8.0320 & 7.1091 & 5.7639 & 5.3290 & 4.9182 & 3.6722 & 3.2958 \\
$v^{2}$ & -3.5062 & -3.4946 & -3.0766 & -3.1474 & -3.1199 & -3.3232 & -3.4860 & -3.6886 \\
$v^{3}$ & 2.5145 & 2.0922 & 2.3948 & 3.8281 & 4.4130 & 4.8964 & 5.0084 & 5.2076 \\
$v d^{2}$ & -3.9006 & -2.8010 & -1.5414 & -0.3353 & 0.2163 & 0.7137 & 0.8194 & 0.6978 \\
$v d^{3}$ & 13.366 & 11.072 & 7.9744 & 4.7691 & 2.9786 & 1.4078 & 0.6490 & 0.3886 \\
$v d^{4}$ & -12.691 & -10.778 & -7.9718 & -5.0841 & -3.3624 & -1.9570 & -1.1804 & -0.8244 \\
$v d^{5}$ & 3.4798 & 3.0006 & 2.2430 & 1.4664 & 0.9881 & 0.6153 & 0.3909 & 0.2820 \\
$v^{2} d$ & 2.8525 & -0.2090 & -2.8546 & -3.8687 & -4.3865 & -4.3728 & -3.8970 & -2.9310 \\
$v^{2} d^{4}$ & 8.8503 & 8.0542 & 5.6164 & 2.9707 & 1.4016 & 0.5671 & 0.2666 & 0.1475 \\
$v^{2} d^{5}$ & -3.2873 & -3.0022 & -2.0892 & -1.1191 & -0.5428 & -0.2404 & -0.1080 & -0.0497 \\
$v^{3} d$ & 0.1346 & 18.169 & 21.588 & 18.895 & 18.515 & 19.504 & 20.912 & 20.944 \\
$v^{3} d^{2}$ & -29.715 & -37.0820 & -27.2990 & -14.8500 & -7.8594 & -4.9007 & -5.1160 & -6.1350 \\
$v^{4} d$ & 14.493 & -7.8908 & -14.526 & -16.995 & -19.729 & -23.103 & -25.878 & -27.462 \\
$v^{4} d^{2}$ & 15.918 & 30.162 & 23.574 & 14.307 & 8.9029 & 6.8545 & 7.3664 & 8.8588 \\
\hline$R^{2}$ & 0.99943 & 0.99947 & 0.99946 & 0.99946 & 0.99953 & 0.99958 & 0.99963 & 0.99965 \\
$\hat{\sigma}_{\epsilon}$ & 0.00881 & 0.00896 & 0.00960 & 0.00995 & 0.00950 & 0.00901 & 0.00856 & 0.00832 \\
\hline & & & & & & & &
\end{tabular}

2. there is a trend break at $\tau$ and detrend both series with knowledge of the actual break location (TB)

We assume that the degree of integration is unknown and estimate the parameter $d$ of the detrended two processes with the Exact Local Whittle (ELW) estimator proposed by Shimotsu (2010) fixing the bandwidth with $m=T^{0.78}$, which is optimal in absence of autocorrelation. With the mean of the maintained two estimates of $d$ we calculate the $5 \%$ critical values by the Gamma distribution obtained by the moments of the response surface regression.

Figure 6 shows the results of this simulation with sample size $T=500$ and 100.000 replications. In the left graphic we vary the break strength parameter $\mu_{2}$ with fixed $\tau=0.5$ and in the right the break location $\tau$ with fixed $\mu_{2}=1$. For (LT) we have the problem, that $d$ can't be estimated anymore consistently and so we have to use heavily biased 

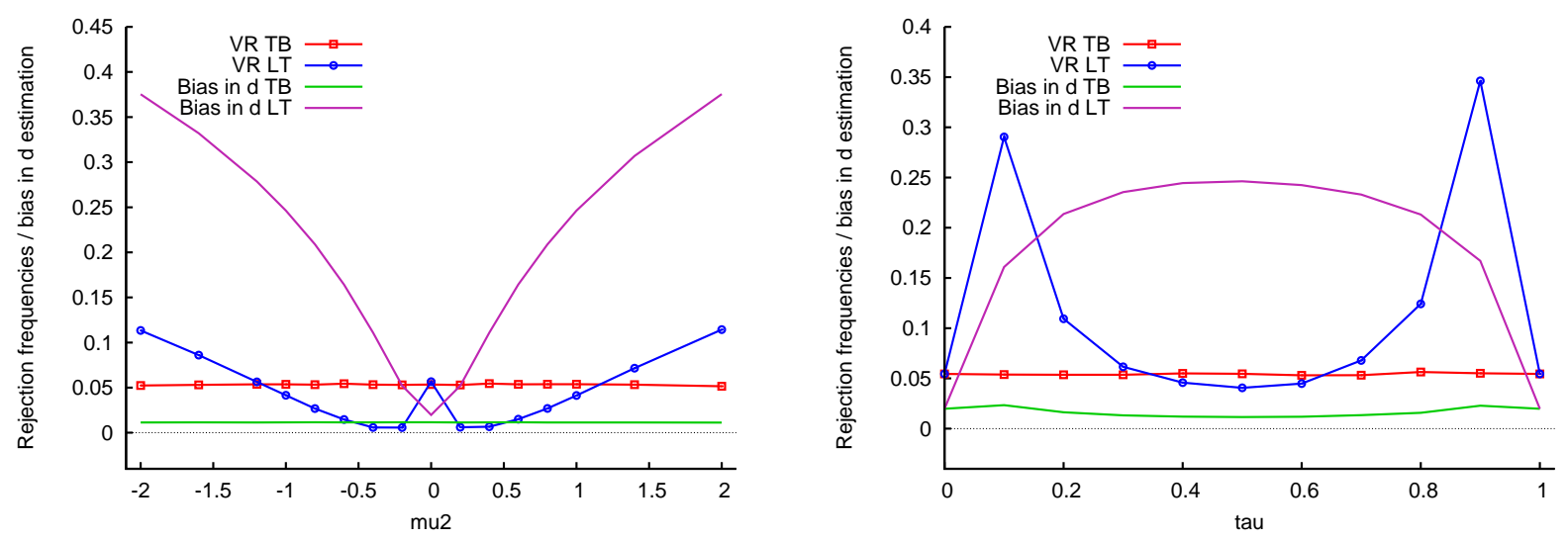

Figure 6: Effect of negligence of a trend break

$d$-estimates to calculate critical values and encounter severely distorted test decisions.

In our next simulation we compare the VR approach with Johansen type rank tests, which takes also trend breaks into account, as proposed by JMN (2000). We chose for the integration parameter the empirical most relevant cases $d=0.8, d=1$ and $d=1.2$. We also want to analyse simple bivariate systems as in our first simulation, but take also into account, that the system might be cointegrated of rank $r=1$ with cointegration strength $b>0$. For the deterministic component of the simulated series we set $\mu_{2}=1$ and $\tau=0.5$ and the integration parameter $d$ is assumed to be known. $\mathrm{VR}_{\text {resp }}$ denotes the rejection frequency of no cointegration using critical values calculated with the response surface parameters reported in table 1 and $2 . \mathrm{VR}_{\text {boot }}$ stands for the decision by using the bootstrapping technique. Therefore, we choose block-of-blocks bootstrapping and did only 10.000 replications as bootstrapping is quite CPU time consumptive. In order to apply the JMN approach we have to determine a lag parameter $k$, which is shown in the index of $\mathrm{JMN}_{k}$. As expected, the power of the $\mathrm{VR}$ approach lacks for $d=0.8$ and seems to be undersized for $T=250$ and $T=500$. The situation gets better for bigger sample sizes like $T=1.000$. The classical approach, which doesn't allow fractional integration, seems to have big problems detecting cointegration relations as it highly rejects the null of no cointegration, when $b=0$. This might be explained, that when the system is integrated of order $d=0.8$, the nonstationarity of the system may often not be detected. So we might expect, that the JMN approach also rejects $r=2$ with a high probability. The power of the JMN approach is better, when $d=1$ and the lag parameter is not highly over-selected. The VR approach has obviously better properties for $d=1.2$. The benefits of bootstrapping exists only for $d=0.8$ and performs actually worse for the other cases.

For the situation with autocorrelated errors (type B) there is a bigger problem with the 
Table 3: Size-corrected power for fractional cointegration models with type A errors

\begin{tabular}{|c|c|c|c|c|c|c|c|c|c|c|}
\hline \multicolumn{6}{|c|}{$T=250$} & \multicolumn{5}{|c|}{$T=500$} \\
\hline$b$ & $\mathrm{VR}_{\text {resp }}$ & $\mathrm{VR}_{\text {boot }}$ & $\mathrm{JMN}_{0}$ & $\mathrm{JMN}_{1}$ & $\mathrm{JMN}_{4}$ & $\mathrm{VR}_{\text {resp }}$ & $\mathrm{VR}_{\text {boot }}$ & $\mathrm{JMN}_{0}$ & $\mathrm{JMN}_{1}$ & $\mathrm{JMN}_{4}$ \\
\hline \multicolumn{11}{|c|}{$d=0.8$} \\
\hline 0 & $1.0 \%$ & $3.3 \%$ & $91.0 \%$ & $62.7 \%$ & $33.3 \%$ & $2.9 \%$ & $4.5 \%$ & $98.7 \%$ & $87.1 \%$ & $58.7 \%$ \\
\hline 0.2 & $14.6 \%$ & $15.8 \%$ & $100.0 \%$ & $96.7 \%$ & $68.3 \%$ & $34.3 \%$ & $27.5 \%$ & $100.0 \%$ & $100.0 \%$ & $96.1 \%$ \\
\hline 0.4 & $68.1 \%$ & $54.2 \%$ & $100.0 \%$ & $100.0 \%$ & $96.3 \%$ & $96.2 \%$ & $87.0 \%$ & $100.0 \%$ & $100.0 \%$ & $100.0 \%$ \\
\hline 0.6 & $98.9 \%$ & $92.3 \%$ & $100.0 \%$ & $100.0 \%$ & $100.0 \%$ & $100.0 \%$ & $100.0 \%$ & $100.0 \%$ & $100.0 \%$ & $100.0 \%$ \\
\hline 0.8 & $100.0 \%$ & $99.9 \%$ & $100.0 \%$ & $100.0 \%$ & $100.0 \%$ & $100.0 \%$ & $100.0 \%$ & $100.0 \%$ & $100.0 \%$ & $100.0 \%$ \\
\hline \multicolumn{11}{|c|}{$d=1$} \\
\hline 0 & $4.3 \%$ & $4.2 \%$ & $5.9 \%$ & $5.9 \%$ & $6.2 \%$ & $5.1 \%$ & $4.8 \%$ & $5.2 \%$ & $5.5 \%$ & $5.3 \%$ \\
\hline 0.2 & $21.7 \%$ & $14.9 \%$ & $52.0 \%$ & $30.1 \%$ & $16.9 \%$ & $26.7 \%$ & $20.8 \%$ & $74.8 \%$ & $47.6 \%$ & $27.1 \%$ \\
\hline 0.4 & $69.5 \%$ & $50.3 \%$ & $99.5 \%$ & $87.1 \%$ & $49.6 \%$ & $84.2 \%$ & $73.3 \%$ & $100.0 \%$ & $99.5 \%$ & $85.6 \%$ \\
\hline 0.6 & $99.3 \%$ & $93.7 \%$ & $100.0 \%$ & $100.0 \%$ & $91.4 \%$ & $100.0 \%$ & $99.8 \%$ & $100.0 \%$ & $100.0 \%$ & $100.0 \%$ \\
\hline 0.8 & $100.0 \%$ & $100.0 \%$ & $100.0 \%$ & $100.0 \%$ & $99.9 \%$ & $100.0 \%$ & $100.0 \%$ & $100.0 \%$ & $100.0 \%$ & $100.0 \%$ \\
\hline 1 & $100.0 \%$ & $100.0 \%$ & $100.0 \%$ & $100.0 \%$ & $100.0 \%$ & $100.0 \%$ & $100.0 \%$ & $100.0 \%$ & $100.0 \%$ & $100.0 \%$ \\
\hline \multicolumn{11}{|c|}{$d=1.2$} \\
\hline 0 & $4.6 \%$ & $4.2 \%$ & $3.2 \%$ & $1.4 \%$ & $1.9 \%$ & $5.3 \%$ & $4.9 \%$ & $5.9 \%$ & $2.2 \%$ & $1.4 \%$ \\
\hline 0.2 & $19.2 \%$ & $12.7 \%$ & $3.1 \%$ & $2.7 \%$ & $3.5 \%$ & $20.6 \%$ & $15.6 \%$ & $4.7 \%$ & $2.6 \%$ & $2.7 \%$ \\
\hline 0.4 & $53.2 \%$ & $37.6 \%$ & $36.8 \%$ & $18.3 \%$ & $10.6 \%$ & $59.8 \%$ & $49.7 \%$ & $62.7 \%$ & $33.6 \%$ & $17.7 \%$ \\
\hline 0.6 & $92.8 \%$ & $83.2 \%$ & $98.6 \%$ & $78.3 \%$ & $39.7 \%$ & $97.5 \%$ & $94.7 \%$ & $100.0 \%$ & $98.7 \%$ & $78.5 \%$ \\
\hline 0.8 & $100.0 \%$ & $99.8 \%$ & $100.0 \%$ & $100.0 \%$ & $86.8 \%$ & $100.0 \%$ & $100.0 \%$ & $100.0 \%$ & $100.0 \%$ & $100.0 \%$ \\
\hline 1 & $100.0 \%$ & $100.0 \%$ & $100.0 \%$ & $100.0 \%$ & $99.8 \%$ & $100.0 \%$ & $100.0 \%$ & $100.0 \%$ & $100.0 \%$ & $100.0 \%$ \\
\hline 1.2 & $100.0 \%$ & $100.0 \%$ & $100.0 \%$ & $100.0 \%$ & $100.0 \%$ & $100.0 \%$ & $100.0 \%$ & $100.0 \%$ & $100.0 \%$ & $100.0 \%$ \\
\hline
\end{tabular}

size than with type A errors. This also vanishes for growing sample sizes, but is still inherent for $T=500$. Bootstrapping helps to solve this problem as the power increases for $d=0.8$ and $d=1$ with low cointegration strengths. For $d=1.2$ it has no positive effect.

We want to close this section with a last Monte Carlo study, when we compare the estimator for the cointegration space defined in (13) with simple OLS estimation. For this simulation we chose also a simple bivariate cointegrated system (excluding the trivial case $b=0$ ) with $d=1$ and $T=250$ fixed. In table 5 we give the bias and root mean squared error of the estimated cointegration space parameters in simulations with 100.000 replications, which shows that OLS is dominant over VR when there is no correlation between the error term of the stochastic trend and the cointegration error (i.e. $\varepsilon_{1, t}$ and 
Table 4: Size-corrected power for fractional cointegration models with type B errors

\begin{tabular}{|c|c|c|c|c|c|c|c|c|c|c|}
\hline \multicolumn{6}{|c|}{$T=250$} & \multicolumn{5}{|c|}{$T=500$} \\
\hline$b$ & $\mathrm{VR}_{\text {resp }}$ & $\mathrm{VR}_{\text {boot }}$ & $\mathrm{JMN}_{0}$ & $\mathrm{JMN}_{1}$ & $\mathrm{JMN}_{4}$ & $\mathrm{VR}_{\text {resp }}$ & $\mathrm{VR}_{\text {boot }}$ & $\mathrm{JMN}_{0}$ & $\mathrm{JMN}_{1}$ & $\mathrm{JMN}_{4}$ \\
\hline \multicolumn{11}{|c|}{$d=0.8$} \\
\hline 0 & $0.0 \%$ & $1.3 \%$ & $1.8 \%$ & $25.0 \%$ & $21.2 \%$ & $0.1 \%$ & $2.0 \%$ & $2.8 \%$ & $49.6 \%$ & $40.4 \%$ \\
\hline 0.2 & $0.1 \%$ & $5.4 \%$ & $10.5 \%$ & $58.1 \%$ & $43.7 \%$ & $1.7 \%$ & $11.8 \%$ & $44.3 \%$ & $93.0 \%$ & $81.7 \%$ \\
\hline 0.4 & $1.7 \%$ & $18.7 \%$ & $78.5 \%$ & $93.8 \%$ & $77.4 \%$ & $24.5 \%$ & $49.5 \%$ & $100.0 \%$ & $100.0 \%$ & $99.7 \%$ \\
\hline 0.6 & $18.8 \%$ & $53.2 \%$ & $100.0 \%$ & $100.0 \%$ & $97.6 \%$ & $89.3 \%$ & $95.1 \%$ & $100.0 \%$ & $100.0 \%$ & $100.0 \%$ \\
\hline 0.8 & $73.3 \%$ & $89.6 \%$ & $100.0 \%$ & $100.0 \%$ & $100.0 \%$ & $100.0 \%$ & $100.0 \%$ & $100.0 \%$ & $100.0 \%$ & $100.0 \%$ \\
\hline \multicolumn{11}{|c|}{$d=1$} \\
\hline 0 & $0.6 \%$ & $2.0 \%$ & $4.2 \%$ & $6.4 \%$ & $6.7 \%$ & $1.6 \%$ & $2.9 \%$ & $4.4 \%$ & $6.0 \%$ & $5.9 \%$ \\
\hline 0.2 & $2.5 \%$ & $6.9 \%$ & $3.1 \%$ & $14.0 \%$ & $12.4 \%$ & $8.2 \%$ & $10.7 \%$ & $3.3 \%$ & $22.2 \%$ & $19.6 \%$ \\
\hline 0.4 & $13.8 \%$ & $21.0 \%$ & $8.1 \%$ & $40.7 \%$ & $30.2 \%$ & $38.9 \%$ & $43.6 \%$ & $28.6 \%$ & $79.8 \%$ & $62.3 \%$ \\
\hline 0.6 & $52.9 \%$ & $54.9 \%$ & $66.9 \%$ & $87.5 \%$ & $65.2 \%$ & $90.4 \%$ & $89.3 \%$ & $99.9 \%$ & $99.9 \%$ & $98.2 \%$ \\
\hline 0.8 & $95.1 \%$ & $92.3 \%$ & $100.0 \%$ & $99.9 \%$ & $95.0 \%$ & $100.0 \%$ & $100.0 \%$ & $100.0 \%$ & $100.0 \%$ & $100.0 \%$ \\
\hline 1 & $100.0 \%$ & $100.0 \%$ & $100.0 \%$ & $100.0 \%$ & $99.9 \%$ & $100.0 \%$ & $100.0 \%$ & $100.0 \%$ & $100.0 \%$ & $100.0 \%$ \\
\hline \multicolumn{11}{|c|}{$d=1.2$} \\
\hline 0 & $2.5 \%$ & $3.2 \%$ & $15.5 \%$ & $3.1 \%$ & $2.9 \%$ & $4.3 \%$ & $4.7 \%$ & $21.0 \%$ & $1.8 \%$ & $1.9 \%$ \\
\hline 0.2 & $7.3 \%$ & $7.4 \%$ & $9.1 \%$ & $4.4 \%$ & $4.1 \%$ & $11.8 \%$ & $12.1 \%$ & $11.9 \%$ & $2.9 \%$ & $3.0 \%$ \\
\hline 0.4 & $21.2 \%$ & $18.5 \%$ & $7.3 \%$ & $10.3 \%$ & $8.8 \%$ & $34.3 \%$ & $34.2 \%$ & $12.2 \%$ & $15.5 \%$ & $12.2 \%$ \\
\hline 0.6 & $53.0 \%$ & $46.6 \%$ & $13.2 \%$ & $35.0 \%$ & $24.0 \%$ & $78.1 \%$ & $75.4 \%$ & $37.1 \%$ & $71.6 \%$ & $53.3 \%$ \\
\hline 0.8 & $92.0 \%$ & $86.0 \%$ & $67.0 \%$ & $83.6 \%$ & $58.7 \%$ & $99.5 \%$ & $99.0 \%$ & $99.8 \%$ & $99.9 \%$ & $97.4 \%$ \\
\hline 1 & $100.0 \%$ & $99.7 \%$ & $100.0 \%$ & $99.8 \%$ & $92.7 \%$ & $100.0 \%$ & $100.0 \%$ & $100.0 \%$ & $100.0 \%$ & $100.0 \%$ \\
\hline 1.2 & $100.0 \%$ & $100.0 \%$ & $100.0 \%$ & $100.0 \%$ & $99.9 \%$ & $100.0 \%$ & $100.0 \%$ & $100.0 \%$ & $100.0 \%$ & $100.0 \%$ \\
\hline
\end{tabular}

$\left.\varepsilon_{2, t}\right)$. If there is a correlation between them, the VR approach is behaving better in terms of bias and RMSE for $b \geq 0.6$. The VR approach has big distortions for low cointegration strength $b$.

\section{Empirical Application}

As an empirical exercise we apply the VR approach to monthly Treasury bill rates covering a time range from December 1958 to December 2011, which contains 637 observations. In figure 7 it can be seen that Tbill rates with three month (M3), six months (M6), one year (Y1) and three years (Y3) have an upward trend to September 1979 and then decrease to the end of the observation period. So we assume exogenously a trend break at $\tau=0.3909$. 
Table 5: Simulation results for estimated cointegration space with $d=1$ and $T=250$ applying VR approach and OLS

\begin{tabular}{|c|c|c|c|c|c|c|c|c|}
\hline \multirow[b]{3}{*}{$b$} & \multicolumn{4}{|c|}{ Type A errors } & \multicolumn{4}{|c|}{ Type B errors } \\
\hline & \multicolumn{2}{|c|}{ VR } & \multicolumn{2}{|c|}{ OLS } & \multicolumn{2}{|c|}{ VR } & \multicolumn{2}{|c|}{ OLS } \\
\hline & Bias & RMSE & Bias & RMSE & Bias & RMSE & Bias & RMSE \\
\hline 0.2 & -0.5191 & 10.413 & -0.0031 & 0.2090 & -0.4612 & 12.414 & -0.0001 & 0.2235 \\
\hline 0.4 & -0.0836 & 3.6442 & 0.0001 & 0.1048 & -0.1364 & 9.0575 & -0.0009 & 0.1122 \\
\hline 0.6 & -0.0066 & 0.0932 & 0.0004 & 0.0561 & -0.0093 & 0.2504 & 0.0009 & 0.0589 \\
\hline 0.8 & -0.0011 & 0.0380 & 0.0002 & 0.0309 & -0.0028 & 0.0425 & -0.0006 & 0.0315 \\
\hline 1 & -0.0004 & 0.0198 & -0.0002 & 0.0181 & -0.0005 & 0.0211 & 0.0000 & 0.0185 \\
\hline \multicolumn{9}{|c|}{ with correlated errors $(\rho=0.75)$} \\
\hline 0.2 & 0.0921 & 15.745 & 0.4133 & 0.4385 & 0.1991 & 25.194 & 0.4010 & 0.4297 \\
\hline 0.4 & 0.0718 & 1.3024 & 0.2225 & 0.2379 & 0.0769 & 1.4689 & 0.2026 & 0.2189 \\
\hline 0.6 & 0.0485 & 0.0714 & 0.1155 & 0.1269 & 0.0430 & 0.1036 & 0.0948 & 0.1060 \\
\hline 0.8 & 0.0167 & 0.0318 & 0.0596 & 0.0682 & 0.0145 & 0.0326 & 0.0385 & 0.0467 \\
\hline 1 & -0.0003 & 0.0146 & 0.0304 & 0.0371 & -0.0003 & 0.0151 & 0.0106 & 0.0179 \\
\hline
\end{tabular}

Looking at the detrended Tbill rates gives us some evidence of mean reverting behavior, which implies an integration parameter $d<1$. Table 6 shows the results of the univariate analysis of the Tbill rates. As a first step we estimate the integration parameter $d$ for the rates by applying the ELW estimator. The estimates don't differ significantly from each other over the reported bandwidths. All estimates are lower than unity and for some bandwidths this seems even to be significant. Applying the univariate VR test for the rates gives also evidence that the rates share an integration parameter lower than 1 . The $p$-values are calculated under the hypothesis $H_{0}: d \geq 1$ with $\tau=0.3909$ and all of them are lower than $5 \%$. Bootstrapping yields exactly the same $p$-values.

For the following multivariate analysis we have to decide which estimate of $d$ we want to use for calculating the $p$-values of the VR rank tests. For that reason we choose the estimates of the lowest bandwidth $m=48$ as the estimated don't differ significantly from the other bandwidths. For every data vector in table 7 we calculate the mean of the estimated $d$ presented in table 6 and use it to determine the $p$-values of the VR statistics. Bootstrapping the VR statistic yields the same results and is therefore left out from the table. For the bivariate case $p=2$ we can mostly confirm the implication on the cointegration rank on a $5 \%$ error level. Surprisingly, for the couple M3 and M6 the hypothesis of $r=2$ can also be rejected on $10 \%$ level. The $p=3$ case is also inline with the theory, while the data vector of M3, Y1 and Y3 have only a significant rejection of 

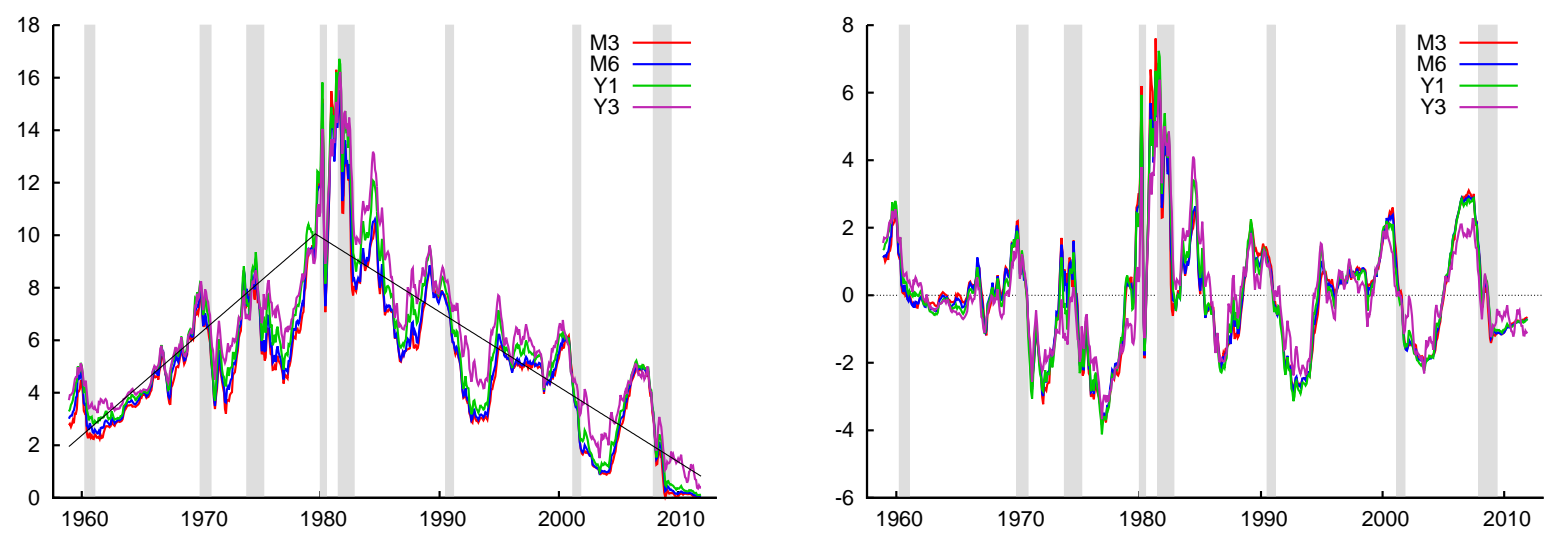

Figure 7: Time series plot with nominal Tbill rates and detrended with a trend break in Sep 79. Phases of recessions are shaded.

Table 6: ELW estimation and univariate VR tests of Tbill rates

\begin{tabular}{c|ccc|cc}
\hline Bandwidth & $m=\left\lfloor T^{0.6}\right\rfloor=48$ & $m=\left\lfloor T^{0.65}\right\rfloor=66$ & $m=\left\lfloor T^{0.7}\right\rfloor=91$ & VR stat & $p$-value \\
\hline M3 & $0.8755^{* *}$ & $0.9071^{*}$ & $0.8729^{* * *}$ & 2.1657 & 0.0110 \\
M6 & $0.9002^{*}$ & $0.8968^{* *}$ & $0.8840^{* *}$ & 2.1575 & 0.0129 \\
Y1 & $0.8883^{*}$ & $0.8817^{* *}$ & $0.8835^{* *}$ & 2.1387 & 0.0186 \\
Y3 & 0.9209 & $0.8816^{* *}$ & $0.9032^{* *}$ & 2.0940 & 0.0410 \\
\hline aSD & 0.0722 & 0.0615 & 0.0524 & & \\
\hline Average & 0.8962 & 0.8918 & 0.8859 & & \\
\hline
\end{tabular}

Note: One, two and three asterisks denote rejection of the hypothesis $H_{0}: d \geq 1$ basing on the ELW estimate of $d$ on $10 \%, 5 \%$ and $1 \%$ error level.

$r=1$ on a $10 \%$ level. This might be explained by the fact of the longest spread time between all involved rates. Finally the full system of $p=4$ including all maturities is also only significant for $r=3$ on a $10 \%$ level, although a weak evidence, but it confirms the implications of the market expectation hypothesis.

Table 8 shows the estimated spaces by applying the VR approach and the strengths estimated by ELW as the difference of the mean integration parameter of the data vector and the estimated cointegration strengths. The errors are produced by multiplying the estimated rotated spaces with the datavectors. Therefore, every row of the reported spaces stands for one cointegration relation. The cointegration strength parameter $b$ is calculated as the difference of the average $d$ of the components of the datavector and the estimated integration order of the cointegration error process by using ELW.

The market expectation hypothesis states, that the cointegration relation itself should be 
Table 7: VR testing for cointegration rank

\begin{tabular}{c|cc|cc|cc|c|cc}
\hline & \multicolumn{3}{|c}{$p-r=1$} & \multicolumn{2}{c}{$p-r=2$} & \multicolumn{2}{c}{$p-r=3$} & \multicolumn{2}{c}{$p-r=4$} \\
Datavector & VR stat & $p$-value & VR stat & $p$-value & VR stat & $p$-value & VR stat & $p$-value \\
\hline (M3, M6) & 2.1570 & 0.0826 & 4.8733 & 0.0000 & - & - & - & - \\
$(\mathrm{M} 3, \mathrm{Y} 1)$ & 2.1364 & 0.1154 & 4.6931 & 0.0001 & - & - & - & - \\
$(\mathrm{M} 3, \mathrm{Y} 3)$ & 2.0872 & 0.1640 & 4.4201 & 0.0070 & - & - & - & - \\
$(\mathrm{M} 6, \mathrm{Y} 1)$ & 2.1258 & 0.1110 & 4.5488 & 0.0010 & - & - & - & - \\
$(\mathrm{M} 6, \mathrm{Y} 3)$ & 2.0712 & 0.1699 & 4.3335 & 0.0161 & - & - & - & - \\
$(\mathrm{Y} 1, \mathrm{Y} 3)$ & 2.0551 & 0.2135 & 4.2737 & 0.0407 & - & - & - & - \\
\hline (M3, M6, Y1) & 2.1099 & 0.1442 & 4.4865 & 0.0037 & 7.2212 & 0.0000 & - & - \\
$(\mathrm{M} 3, \mathrm{M} 6, \mathrm{Y} 3)$ & 2.0286 & 0.2873 & 4.2611 & 0.0541 & 6.9825 & 0.0000 & - & - \\
$(\mathrm{M} 3, \mathrm{Y} 1, \mathrm{Y} 3)$ & 2.0229 & 0.3116 & 4.2246 & 0.0869 & 6.8424 & 0.0001 & - & - \\
$(\mathrm{M} 6, \mathrm{Y} 1, \mathrm{Y} 3)$ & 2.0434 & 0.2422 & 4.2458 & 0.0580 & 6.7670 & 0.0002 & - & - \\
\hline (M3, M6, Y1, Y3) & 2.0180 & 0.3206 & 4.2186 & 0.0897 & 6.7267 & 0.0006 & 9.4736 & 0.0000 \\
\hline
\end{tabular}

the spreads between the different maturities. So, the coefficients of the rows of the rotated spaces should add up to zero. The estimated spaces itself differ from the expected form, which might be explained by the reason that the cointegration strength is lower when the spread time gets longer and therefore we face higher variances in estimating the spaces.

\section{Conclusion}

In this paper we presented response surface parameters to determine critical values and pvalues for the VR test of fractional (co-)integration, when the underlying processes inhibit a linear trend with the possibility of a structural break. Further work might be done to take a second break into account. Modeling more than two breaks in empirical analysis might not be relevant as it is difficult to determine and to test. More interesting can be the approach by Johansen and Nielsen (2011), who stated a fractional error correction model, which also allows to test restrictions on the cointegration space. Practitioners should also be aware that the order of fractional integration might not be stable over a longer time. So models, which can take a changing $b$ into account might be interesting and opens a wide field for further research. 
Table 8: Estimated cointegration spaces and strengths

\begin{tabular}{|c|c|c|c|c|}
\hline \multirow[b]{2}{*}{ Datavector } & \multirow{2}{*}{$\begin{array}{c}\text { estimated rotated space } \\
\qquad \hat{\boldsymbol{\beta}}^{\prime}\end{array}$} & \multicolumn{3}{|c|}{ estimated strength $\hat{b}$} \\
\hline & & $m=48$ & $m=66$ & $m=91$ \\
\hline$(\mathrm{M} 3, \mathrm{M} 6)$ & $1-1.0245$ & 0.5454 & 0.6021 & 0.4692 \\
\hline (M3, Y1) & $1-0.9565$ & 0.4029 & 0.4539 & 0.3326 \\
\hline (M3, Y3) & $1-0.9433$ & 0.1902 & 0.1991 & 0.1829 \\
\hline$(\mathrm{M} 6, \mathrm{Y} 1)$ & $1-0.9256$ & 0.1962 & 0.3236 & 0.2385 \\
\hline (M6, Y3) & $1-0.8620$ & 0.0893 & 0.1152 & 0.1164 \\
\hline$(\mathrm{Y} 1, \mathrm{Y} 3)$ & $\left(\begin{array}{ll}1 & -0.9134\end{array}\right)$ & 0.0811 & 0.0816 & 0.0976 \\
\hline \multirow{2}{*}{$(\mathrm{M} 3, \mathrm{M} 6, \mathrm{Y} 1)$} & $\left.\begin{array}{lll}1 & 0 & -0.9343\end{array}\right)$ & 0.3816 & 0.4304 & 0.3218 \\
\hline & $\begin{array}{lll}0 & 1 & -0.9174\end{array}$ & 0.1803 & 0.3145 & 0.2272 \\
\hline \multirow{2}{*}{ (M3, M6, Y3) } & $\begin{array}{lll}1 & 0 & -0.7975\end{array}$ & 0.1520 & 0.1435 & 0.1459 \\
\hline & $\begin{array}{lll}0 & 1 & -0.7861\end{array}$ & 0.0635 & 0.0951 & 0.0925 \\
\hline \multirow{2}{*}{ (M3, Y1, Y3) } & $\begin{array}{lll}1 & 0 & -0.7584\end{array}$ & 0.1364 & 0.1225 & 0.1352 \\
\hline & $\begin{array}{ccc}0 & 1 & -0.8609\end{array}$ & 0.0654 & 0.0760 & 0.0797 \\
\hline \multirow{2}{*}{$(\mathrm{M} 6, \mathrm{Y} 1, \mathrm{Y} 3)$} & $\begin{array}{lll}1 & 0 & -0.7710\end{array}$ & 0.0651 & 0.0817 & 0.0926 \\
\hline & $\left(\begin{array}{lll}0 & 1 & -0.8830\end{array}\right)$ & 0.0762 & 0.0783 & 0.0880 \\
\hline \multirow{3}{*}{ (M3, M6, Y1, Y3) } & $\left(\begin{array}{cccc}1 & 0 & 0 & -0.7587\end{array}\right)$ & 0.1378 & 0.1243 & 0.1346 \\
\hline & $\begin{array}{llll}0 & 1 & 0 & -0.7470\end{array}$ & 0.0541 & 0.0794 & 0.0830 \\
\hline & $\begin{array}{llll}0 & 0 & 1 & -0.8645\end{array}$ & 0.0671 & 0.0786 & 0.0798 \\
\hline
\end{tabular}

\section{References}

[1] Breitung, J., Hassler, U. (2002): Inference on the cointegration rank in fractionally integrated processes. Journal of Econometrics 110, 167-185.

[2] Campbell, J. Y., Shiller, R. J. (1987): Cointegration and tests of present value models. Journal of Political Economy 95, 1062-1088.

[3] Doornik, J. A. (1998): Approximations to the Asymptotic Distribution of Cointegration Tests. Journal of Economic Surveys 12, 573-593.

[4] Engle, R. F., Granger, C. W. J. (1987): Co-Integration and error correction: Representation, estimation and testing. Econometrica 55, 251-276.

[5] Engsted, T., Tanggaard, C. (1994): Cointegration and the US term structure. Journal of Banking and Finance 18, 167-181. 
[6] Hall, A. D., Anderson, H. M., Granger C. W. J. (1992): A cointegration analysis of treasury bill yields. Review of Economics and Statistics 74, 116-126.

[7] Hosking, J. R. M. (1980): Fractional differencing. Biometrika 68, 165-176.

[8] Iacone, F. (2009): A semiparametric analysis of the term structure of the US interest rates. Oxford Bulletin of Economics and Statistics 71, 475-490.

[9] Johansen, S., Mosconi, R., Nielsen, B. (2000): Cointegration analysis in the presence of structural breaks in the deterministic trend. Econometrics Journal 3, 216-249.

[10] Johansen, S. (2008): A representation theory for a class of vector autoregressive models for fractional processes. Econometric Theory 24, 651-676.

[11] Johansen, S., Nielsen, M. Ø. (2011): Likelihood inference for a fractionally cointegrated vector autoregressive model. Working Paper.

[12] Marinucci, D., Robinson, P. M. (1999): Alternative forms of fractional Brownian Motion. Journal of Statistical Planing and Inference 80, 111-122.

[13] Nielsen, M. Ø., Shimotsu, K. (2007): Determining the cointegrating rank in nonstationary fractional systems by the exact local Whittle approach. Journal of Econometrics $141,574-596$.

[14] Nielsen, M. Ø. (2008): A powerful test of the autoregressive unit root hypothesis based on a tuning parameter free statistic. Econometric Theory 25, 1515-1544.

[15] Nielsen, M. Ø. (2010): Nonparametric cointegration analysis of fractional systems with unknown integration orders. Journal of Econometrics 155, 170-187.

[16] Perron, P. (1989): The great crash, the oil price shock, and the unit root hypothesis. Econometrica 57, 1361-1401.

[17] Phillips, P. C. B., Ouliaris, S. (1988): Testing for cointegration using principal components methods. Journal of Economic Dynamics and Control 12, 205-230.

[18] Robinson, P. M. (1995): Gaussian semiparametric estimation of long range dependence. Annals of Statistics 23, 1630-1661.

[19] Robinson, P. M., Yajima, Y. (2002): Determination of cointegrating rank in fractional systems. Journal of Econometrics 106, 217-241. 
[20] Shimotsu, K., Phillips, P.C.B (2006): Local Whittle estimation of fractional integration and some of its variants. Journal of Econometrics 130, 209-333.

[21] Shimotsu, K. (2010): Exact local Whittle estimation of fractional integration with unknown mean and time trend. Econometric Theory 26, 501-540. 